\title{
The L1-dependant and Pol III transcribed Alu retrotransposon, from its discovery to innate immunity
}

\author{
Ludwig Stenz ${ }^{1,2}$ (D) \\ Received: 8 January 2021 / Accepted: 26 February 2021 / Published online: 16 March 2021 \\ (c) The Author(s) 2021
}

\begin{abstract}
The 300 bp dimeric repeats digestible by AluI were discovered in 1979. Since then, Alu were involved in the most fundamental epigenetic mechanisms, namely reprogramming, pluripotency, imprinting and mosaicism. These Alu encode a family of retrotransposons transcribed by the RNA Pol III machinery, notably when the cytosines that constitute their sequences are de-methylated. Then, Alu hijack the functions of ORF2 encoded by another transposons named L1 during reverse transcription and integration into new sites. That mechanism functions as a complex genetic parasite able to copy-paste Alu sequences. Doing that, Alu have modified even the size of the human genome, as well as of other primate genomes, during 65 million years of co-evolution. Actually, one germline retro-transposition still occurs each 20 births. Thus, Alu continue to modify our human genome nowadays and were implicated in de novo mutation causing diseases including deletions, duplications and rearrangements. Most recently, retrotransposons were found to trigger neuronal diversity by inducing mosaicism in the brain. Finally, boosted during viral infections, Alu clearly interact with the innate immune system. The purpose of that review is to give a condensed overview of all these major findings that concern the fascinating physiology of Alu from their discovery up to the current knowledge.
\end{abstract}

Keywords Alu $\cdot$ Retrotransposons $\cdot$ DNA methylation $\cdot$ Reprogramming $\cdot$ Pluripotency $\cdot$ Imprinting $\cdot$ Mosaicism

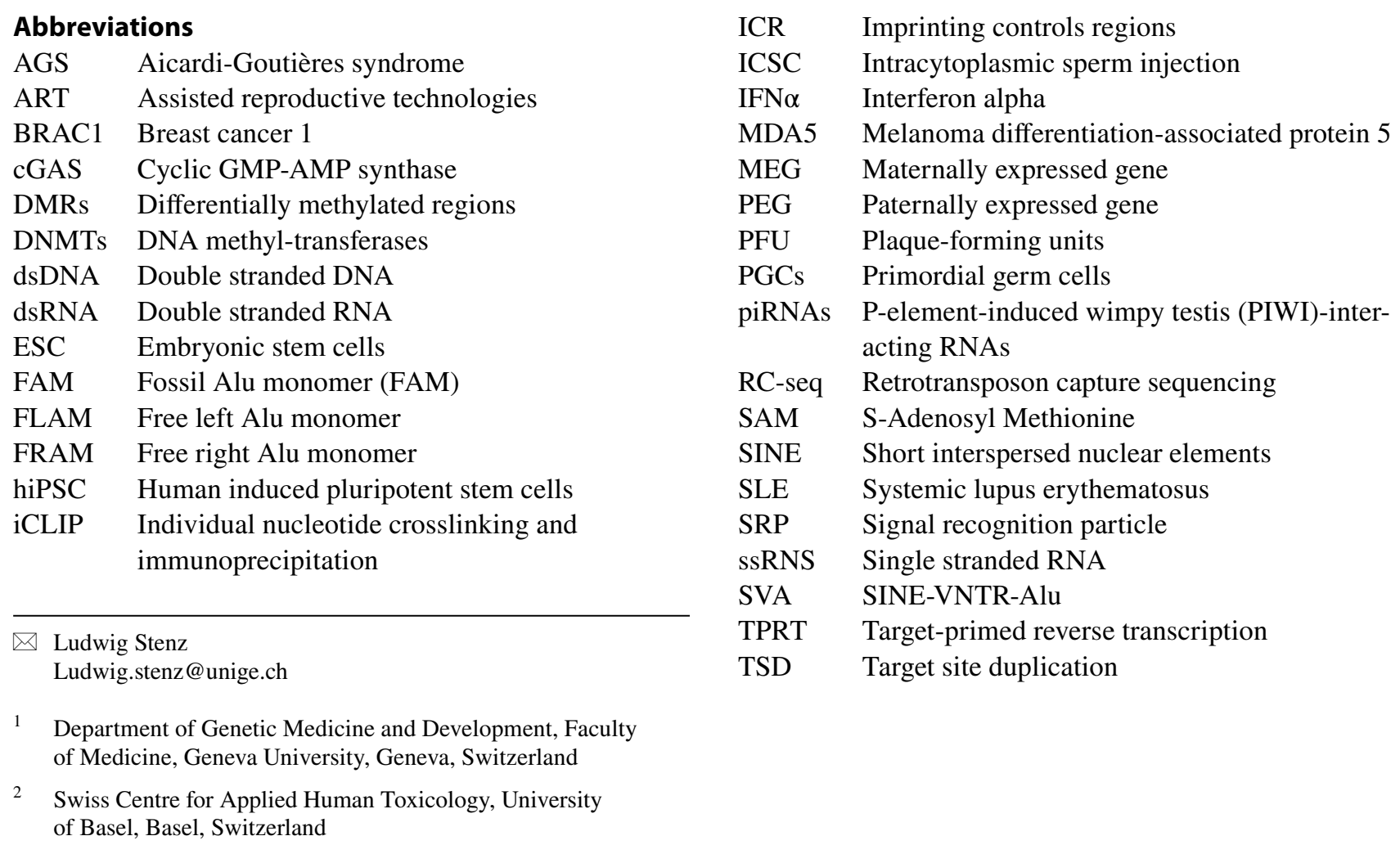




\section{Background}

Houck $\mathrm{C}$ et al.discovered and characterized the Alu repeats in 1979 [1]. Briefly, the authors isolated DNA from human placenta and radio-iodinated it. Using a DNA re-association kinetics technic called $\mathrm{C}_{\mathrm{o}} \mathrm{t}$, the DNA was denatured by boiling before partial renaturation at $60{ }^{\circ} \mathrm{C}$. The remaining single stranded DNA was digested by $\mathrm{S} 1$ nuclease. The authors found a clear enrichment of 300 base pairs (bp) long DNA duplex. They wished to determine whether these repeated elements belong to one family of sequences. They purified these $300 \mathrm{bp}$ repeats and digested it with restriction enzymes. AluI from Arthrobacter luteus bacterium cuts AGCT and producing blunt ends in a methylation insensitivity manner. $A l u \mathrm{I}$ was able to produce two bands of 170 and 120 bp digesting "at least half" of these duplexes, therefore, the authors named these duplex "Alu" and conclude that they should belong to the same family of highly repeated sequences. They also reported that Alu represent 5\% of the human genomic mass. Today, we know that multiple copies of Alu are dispersed into the human genome representing more than $10 \%$ of it (Table 1), whereas $99 \%$ of each Alu repeats consist of unique sequences [2]. The discovery of the Alu repeats raised questions concerning both a putative function and their origin.

\section{Hypothetical origin}

\section{Alu repeats derived from the 7SL RNA via a fossil Alu monomer (FAM)}

Briefly, 7SL RNA (encoded in Human by the 299 bp RN7SL1 gene) is the RNA component of a universally conserved ribonucleoprotein complex, named the signal recognition particle (SRP). SRP is essential for translocation of secreted proteins. The cloning of the 7SL RNA highlights a high homology between the $5^{\prime}$ end of 7SL RNA and the Alu DNA repeats [7]. Later on, broader sequence comparison revealed that the 7SL RNA consists in an Alu monomeric sequence interrupted by a $155 \mathrm{bp}$

Table 1 Representation of Alu sequences in the human genome

\begin{tabular}{llll}
\hline Alu number & Alu Gb & $\begin{array}{l}\text { \% of haploid } \\
\text { genome }\end{array}$ & References \\
\hline$>500,000$ & 0.15 & 4.5 & {$[3]$} \\
923,277 & 0.28 & 8.4 & {$[2]$} \\
965,000 & 0.29 & 8.8 & {$[4]$} \\
$1,100,000$ & 0.33 & 10 & {$[5]$} \\
$1,500,000$ & 0.45 & 13.6 & {$[6]$} \\
\hline
\end{tabular}

long and 7SL RNA specific sequence [8]. That observation supports the hypothesis that an Alu monomer derived from a deletion of the central part of the 7SL RNA. Further, an 11 bp residual sequence (5'-TGTGAATAGCC-3') of the 7SL gene localized specifically in the free right Alu monomer (FRAM) and not in the free left Alu monomer (FLAM). For that reason, geneticists thought that the FRAM should precede the FLAM. Phylogenic analyses were compatible with the hypothesis that a 7SL RNA gives rise first to a fossil Alu monomer (FAM) as a common ancestor for short interspersed nuclear elements (SINE). Then, the FAM gives rise to the primate-specific FRAM and FLAM before they fused together, giving rise to a dimeric mobile Alu [9]. Dimeric Alu propagate in the genome during 65 million years of evolution [10] representing today the most abundant repeats found in the human genome [11], see Table 1.

\section{Alu families}

Alu repeats are subdivided in three families due to different consensus sequences and different times of appearance in the human lineage. Mutation densities were used to estimate the approximate age of each family [12]. These families were originally discovered by restriction digestion and named differently across the bibliography such as "PV", "precise", "major" [13], before a consensual nomenclature ("Y", "S", "J") was adopted [14]. Alu families were further divided into subfamilies based on comparisons between aligned Alu sequences revealing high heterogeneity of certain positions (Table 2).

The first 65 million years old AluJ family is the oldest one, encompass inactive Alu repeats, meaning these repeats lost retro-transposition potential due to the accumulation of mutations. The first subdivision thus segregates that AluJ family, more similar to 7SL RNA than the AluS family, itself subdivided afterwards [18]. The second AluS family is 30 million years old and still contains some active Alu. The third AluY family is the most active, aged between 2 and 4 million years old [19]. The Yb subfamily of the AluY family seems the most actively evolving nowadays [12]. Currently, more than 6000 Alu retrotransposons are active per human genome and belong to $\mathrm{Y}$ and $\mathrm{S}$ subfamilies [5].
Table 2 Alu retro-transposition rates per births

\begin{tabular}{ll}
\hline $\begin{array}{l}\text { Alu insertion per } \\
\text { birth }\end{array}$ & References \\
\hline $1 / 200$ & {$[3]$} \\
$1 / 40$ & {$[15]$} \\
$1 / 20$ & {$[16]$} \\
$1 / 21$ & {$[17]$} \\
\hline
\end{tabular}




\section{Alu retro-transposition}

It is accepted today that the high number of Alu repeats has increased due to retrotransposition. Alu retrotransposition is a "copy and paste" process, altering the structure of the human genome, driving genomic variations and causing inheritable genetic diseases. Retrotransposition implies transcription, as well as an endonuclease activity to cleave the future integration site and a reverse transcriptase combined with an integrase activity to produce the cDNA and to insert it within a new site.

Integration of Alu occurs in the canonical L1 endonuclease recognition sequence "TTAAAAA" [20]. An "A" box (TGGCTCACGCC) and a "B" box (GTTCGAGAC) are present within the internal promoter of numerous Alu repeats recognized by the TFIIIC and TFIIIB, triggering RNA PolIII mediated transcription; see Fig. 1 [21, 22]. ORF2 protein encoded by another transposon named L1 carries both endonuclease and reverse transcriptase domains required for Alu retrotransposition [23]. Alu reverse transcription is initiated directly at the target locus after cleaving genomic DNA with target-primed reverse transcription (TPRT) or alternative mechanisms [24].

Functional study on Alu retrotransposition was limited since a French group specialized in the study of retro-transposon was able to generate a neo ${ }^{\text {tet }}$ construct into an Alu. First, they inserted the Tetrahymena thermophila 23S rRNA group I intron (tet) within a neomycin-containing cassette (neo). The tet intron is auto-catalytic, once transcribed, it can self-splice in vitro without the requirement of cellular components. In addition, the splicing out of the intron restores the neomycin-coding sequence. They introduced the construct within a vector itself transduced in infected cells some becoming resistant to the G418 antibiotic. That assay was able to recognize $\mathrm{G} 418^{\mathrm{R}}$ cells in which the splicing occurs [26].

In 2003, the same group introduced the neo ${ }^{\text {tet }}$ construct separately into both polyA regions of the Alu sequence located within the NF1 gene that was found in a patient suffering from neurofibromatosis. The neo ${ }^{\text {tet }}$ marked Alu was introduced thereafter between the enhancer and the transcription terminator of the Alu-like 7SL RNA. This marked Alu was then used to measure Alu mobilization. Indeed, the neo cassette becomes functional only after a cycle of retrotransposition, i.e. one cycle of transcription, reverse transcription and integration. Transposition was thus quantifiable by counting the number of $\mathrm{G} 418^{\mathrm{R}}$ clones. The assay demonstrates that the marked Alu was able to retro-transpose itself actively in the human genome when co-transduced with an expression vector for LINE proteins essentially ORF2. In addition, Alu insertions identified by invert PCR occur across different chromosomes and the presence of the second poly-A tail was required for Alu retro-transposition.
Redundant sequences sandwiches the inserted Alu due to target site duplication (TSD). The authors demonstrated LINE-ORF2 mediated retro-transposition of marked Alu [27]. Thus, convincing results demonstrate that Alu retrotransposition is not autonomous: Alu parasite the L1 retrotransposition machinery to achieve their own replication. Therefore, Alu may be seen as a parasite's parasite [28].

\section{Clinical consequences of ALU retrotransposition}

Alu insertion may happen de novo. Depending on the insertion site, the mutation may affect human health. In 1991, Wallace et al. reports the first known de novo Alu insertion discovered by Sanger sequencing of PCR products in a patient affected by neurofibromatosis of type 1 , a rare genetic disease with increased risk for tumor formation in the nervous tissues. The insertion occurred within an intron located between exon 5 and 6 of the NF1 gene. The authors thought that the Alu insertion altered the splice site by affecting the branch-point recognition process. One NF1 copy produced a shortened mature RNA lacking exon 6. That Alu insertion occurred de novo, it was absent in the DNA of both the mother and the father [29]. This Alu belongs to the youngest $\mathrm{Y}$ family and was functional for transposition as demonstrated decades after with the development of the Alu mobilization assay previously described. Two years after, a second de novo pathogenic Alu insertion was discovered in a patient suffering from severe haemophilia B. That time, the insertion of Alu interrupted the reading frame within the exon V of the Factor IX gene, resulting in a premature stop codon at glutamic acid 96 [30]. These two reports demonstrate together that insertions of Alu in either introns or exons may happen de novo and may cause diseases. The insertions most probably affected the germ cells, otherwise it would create mosaicism, as explained later.

In addition to pathogenic insertion by retrotransposition, Alu repeats concentrated in or near genes may trigger gene deletion or duplication by homologous recombination notably during crossing-over. In such cases, the retrotransposition of Alu is not required to cause the pathogenic events, but was required to create such Alu-rich regions in genes coding sequences. For example, Alu-mediated deletion by homologous recombination of the $\alpha$-globin gene was implicated in $\alpha$-thalassemia [31], whereas a duplication of a $14 \mathrm{~kb}$ sequence encompassing exons 2 through exon 8 in the LDL receptor gene produced a 50,000 Daltons larger LDL receptor in a patient suffering hypercholesterolaemia [32]. A worthy example concerns the abnormally high concentration of Alu repeats (41.5\%) found within introns of the hyper-variable breast cancer 1 (BRCA1) locus. BRAC1 genotyping is used as a breast cancer predictor. Recently, the authors established that Alu may well be responsible for mediating BRCA1 gene rearrangements in patient's tumors [33]. 
A

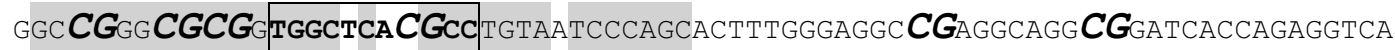

GGA GTT CGAGAC CAGCCTGGCCAACATGGTGAAACCC $\boldsymbol{C}$ TCTCTACTAAAAAATTTAAAAAAAAAAAAAAA T TAGC

$\boldsymbol{C G}_{\mathrm{GG}} \boldsymbol{C} \boldsymbol{G}_{\mathrm{TGGTGG}} \boldsymbol{C} \boldsymbol{G}_{\mathrm{GG}} \boldsymbol{C} \boldsymbol{G} \mathrm{C}$

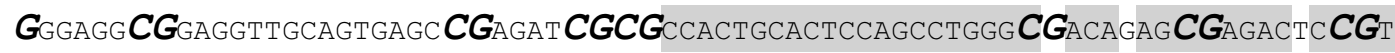

CTCAAAAAAAAAAAAAAAAAAAAAAAAAAAAAAAAA

B

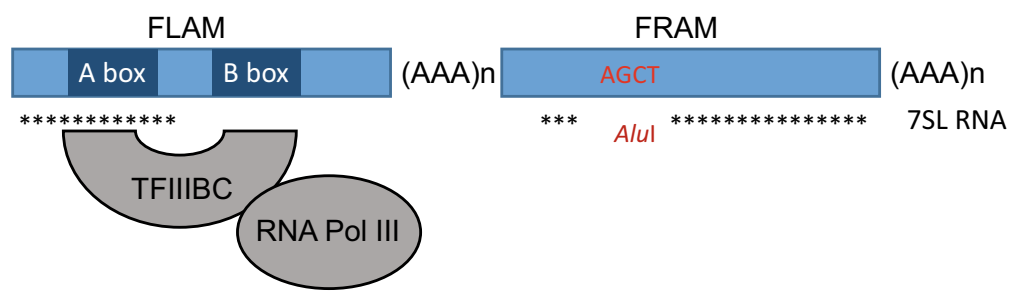

C

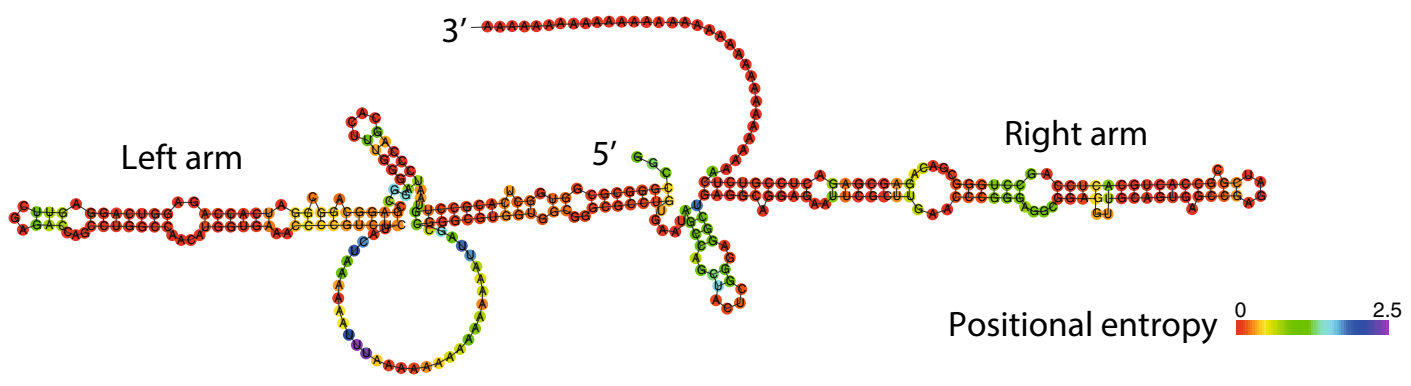

D

L1 ORF2
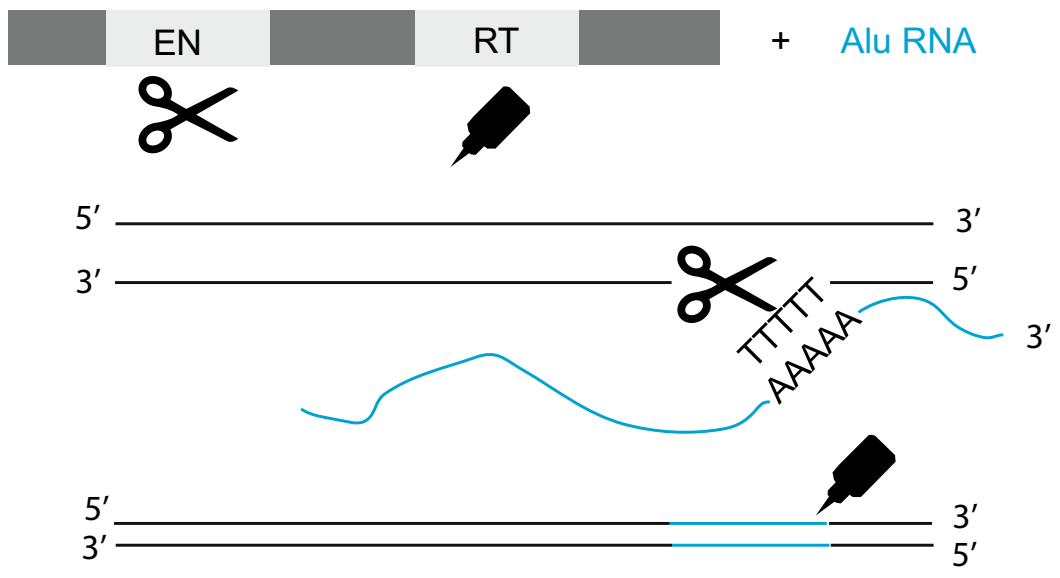

A reference review summarized the different clinical consequences of Alu and estimated that Alu contribute at least in $0.3 \%$ of all human genetic diseases [3]. Alu were responsible for insulin-resistant type II diabetes, Lesch-Nyhan syndrome, Tay-Sachs disease, complement component C3 deficiency, familial hypercholesterolaemia and several types of cancer including Ewing sarcoma, breast cancer and acute myelogenous leukaemia (see review). The contribution of 
४Fig. 1 Alu illustrative sequence and retrotransposition. A A 328 $\mathrm{pb}$ long sequence representing an Alu that hits by blast at numerous positions in Homo sapiens, Pan troglodytes and Gorilla gorilla genomes. AGCT: $A l u \mathrm{I}$ restriction site characterizing the repeats as deriving from one family at their discovery giving them the name "Alu". $\boldsymbol{C G}$ : $\mathrm{CpG}$ sites in which the cytosines may be methylated appear in bold italic with an increased size of the font. Here 22 $\mathrm{CpG}$ sites are present in that Alu illustrative sequence. Highlighted in gray, homologous sequences with the RN7SL1 gene encoding 7SL RNA thought to be the origin of the Alu. The TGTGAA TAGCC 11 bp residual sequence of the 7SL gene localized specifically in the free right Alu monomer (FRAM), showed both underlined and highlighted. Evolutionary, the FRAM precede the FLAM. TGGCTCACGCC = A box, GTTCGAGAC = B

box, both recognized by TFIIIBC and located in the first Alu monomer. These boxes promote transcription by RNA Pol III. Both contain a CpG site. AAAAAATTTAAAAAAAAAAAAAAA: the first polyA tail separating both Alu monomers. AAAAAAAAAAAAAAAA AAAAAAAAAAAAAAAAAA: the second poly-A tail which length may be proportional to transposition efficiency. $\mathbf{B}$ Transcription of Alu by RNA Pol III. TFIIIBC binds to A and B box in the FLAM of Alu. The stars represent the positions conserved between Alu and the 7SL RNA. C Predicted secondary structure of Alu RNA with the left and right arms as well as its 3' poly-A tail. The structure was predicted using the Vienna RNA website [25]. Note that proteins bind to Alu RNA, not shown. D transposition of Alu initiated with an Alu RNA and the L1 ORF2. L1 ORF2 contains an endonuclease domain (EN) symbolized as scissors and a reverse transcriptase domain (RT) symbolized as a glue. EN cuts the DNA at T-rich sequence, allowing the binding of the Alu 3' poly-A tail. Reverse transcription of Alu RNA to cDNA is done by RT. Note that the second DNA strand is cut and the second Alu cDNA strand is synthesized resulting in a new Alu element in the genome flanked by short direct repeats, not shown

Alu in the etiology of various human diseases is currently expanding. Alu retrotransposons contribute to at least 37 neurological and neurodegenerative diseases, mainly affecting mitochondrial functions [34]. Finally, the high pathogenic potential of Alu suggests the existence of cellular protective mechanisms.

\section{Epigenetic repression of Alu transposition}

Epigenetic mechanisms silencing Alu to prevent retro-transposition were rapidly suspected to be required to protect the genome. It was estimated that $99 \%$ of Alu may be silenced resulting in 100 to 1000 cytoplasmic Alu transcripts per cell [35] from around 1 million of Alu DNA copies [5]. Thus, a very low level of Alu transcription occurs at less than 0.001 transcripts per Alu copy in a cell. Among the known epigenetic mechanisms, DNA methylation was rapidly suggested to prevent Alu transcription [36]. DNA methylation implies the addition of a methyl group to cytosine, located within $\mathrm{CpG}$ dyads in mammals. DNA methylation is tough to package Alu into an inactive chromatin structure denying access of essential transcription factors, notably TFIIIB, TFIIIC and RNA Pol III. Lack of Alu transcription should prevent their transposition. Numerous lines of evidence determined that
DNA methylation indirectly controls Alu transpositions by silencing Alu transcription.

The first line of functional evidence derived from HeLa cells treated with $8 \mu \mathrm{M}$ 5-azacytidine, an inhibitor of the DNA methyltransferases. The treatment resulted in hypomethylation of Alu, detected by dot blot of DNA after digestion with the methylation sensitive BstUI restriction enzyme. In addition, increased Alu transcripts after the 5-azacytidine treatment was shown with primer extension and northern blot [35]. Finally, cDNA cloning and sequencing allowed the authors to conclude that all families of Alu were transcribed by Pol III In vivo after de-methylation induced by the 5-azacytldine treatment in the HeLa human cells.

The second line of evidence concerns the composition and the status of the $\mathrm{CpG}$ sites localizes within Alu. Approximately 20 to $24 \mathrm{CpG}$ sites able to be methylated are present per $300 \mathrm{bp}$ long Alu sequences. This represents a ninefold excess of $\mathrm{CpG}$ as compared with the genome. In fact, $23 \%$ of $\mathrm{CpG}$ in the genome are localized within an Alu ( 7 millions per 30 millions). Moreover, these $\mathrm{CpG}$ within Alu have been found heavily methylated in numerous studies (9), whereas hypo-methylation of Alu associates with cancers and tumors [37]. Interestingly, $\mathrm{CpG}$ residues in the youngest Alu subfamilies, which lost the retro-transposition activity, are largely methylated in vivo [13].

The third line of evidence concerns the study across organisms revealing that the organisms containing the CpG methylation machinery can tolerate genomic expansion driven by Alu. Interestingly, these organisms present a decreasing proportion of $\mathrm{CpG}$ dinucleotides over evolutionary time [38]. That paper suggests that methylation in transposons is a prerequisite for genomic expansion, whereas associated GC depletions occurs because the methylated cytosine had increased chance of being mutated into a thymine by uncorrected spontaneous deamination. Thus, during evolution, Alu integration followed by methylation may well produce GC poor extra-DNA. This GC poor extra-DNA seems to be used by the host for genomic regulatory purposes [38]. Indeed, the extra DNA can supply the host with new promoters, enhancers, insulators and may thus establish novel gene regulatory networks that may confer an evolutionary advantage in particular environmental conditions.

Methylation mediated repression of Alu was and remains consensually accepted until the two Varshney et al.publications on the modifications in histones in Alu using chromatin immunoprecipitations based techniques $[39,40]$. The authors claim that methylation at lysine 9 of histone $\mathrm{H} 3$ rather than DNA methylation prevents Alu transcription, whereas methylation of Alu prevents translocation. The authors did not find elevation of Alu transcription after treating HeLa and fibroblast cells with $4 \mu \mathrm{M}$ 5 -azacytldine, a twofold decreased concentration as compared with the Liu et al. paper. Increased Alu transcription 
and RNA Pol III loading were observed after treating cells with a selective inhibitor of the SUV39 methyltransferases named chaetocin, the SUV39 being responsible for histone methylation. However, the most probable scenario seems that both DNA methylation and histone modification cooperate together to prevent both Alu transcription and transposition

\section{Hypomethylated Alu in cancers}

In theory, global Alu hypomethylation may increase the risk of cancer by promoting genomic instability, notably if Alu retrotransposition is poorly prevented. Another hypothesis implies Alu mediated expression of dominant oncogenes. In addition, as methylated $\mathrm{CpG}$ are more prone to mutation and concentrated within Alu, some of these mutations may also trigger cancers. In contrast, hypermethylated Alu located in the promoter of recessive tumor suppressors, which appeared enriched in Alu repeats [41], may also increase the risk of cancers, by interfering though silencing or even rearrangement, such as in the p53 [42], with an important mechanism of defense. Interestingly, the first human oncogene (human bladder carcinoma (EJ) oncogene) was discovered using an Alu marker-rescue experiment [43]. Today, the methylation status of Alu repeats was studied in various cancer types as a putative contributor to cancer etiologies. In a recent meta-analysis including 2719 cancer cases and 3018 controls, the conclusion was that hypomethylation of Alu occurs in carcinoma [44].

\section{Hypomethylated Alu in aging}

Aging is a timeline natural process with increased cellular senescence, risks of degenerative diseases and deaths. Currently, aging at the cellular and organism levels may well be epigenetically encoded. Indeed, the Horwath epigenetic clock takes measures of DNA methylation at specific $\mathrm{CpG}$ sites to successfully predict ages [45], whereas the genomic hypomethylation hypothesis of aging postulates that the global level of DNA methylation decreased with ages [46]. It would be interesting to assess the proportion of $\mathrm{CpG}$ sites incorporated within the epigenetic clock that belongs to Alu repeats, whereas methylation of Alu repeats is already a good surrogate of the entire epigenome [47]. In that aging context, possible roles of human Alu elements in aging could be important [48]. Indeed, Alu are an endogenous source of genomic instability and thus may well promote contribute to lifespan variation. Epigenetic controls of Alu required enzymatic activities (DNMTs) whose efficiency may decrease during aging, notably for DNMT1 in aging fibroblasts [49].

\section{Alu and reprogramming}

Reprogramming is a dynamic genome-wide erasure of DNA methylation marks followed by the establishment of new ones [50]. Reprogramming may theoretically trigger mobilization of all transposable elements that are usually kept silent inside compacted and methylated DNA such as L1-dependant Alu retrotransposons. Mobilization of Alu represents a high risk for the maintenance of the genomic integrity. Therefore, the epigenetic status of transposable elements as well as the identification of protecting mechanisms preventing detrimental transpositions of Alu during reprogramming are of high concern.

Naturally, reprogramming is linked to the sexual reproduction and occurs in two distinct "waves" affecting the germ cell lineage, conferring extensive developmental potential. The first reprogramming wave begins in the zygote, just after the fertilization and confers totipotency, meaning the ability to develop itself into all specialized cells forming an organism. The reprogrammed zygote gives rise to the pluripotent embryonic stem cells (ESC) able to differentiate into the three known germ layers named endoderm, mesoderm and ectoderm [51]. The second reprogramming wave occurs during the fetal life in the primordial germ cells (PGCs). In contrast to the zygote, PGCs remain highly specialized and unipotent, as being the progenitors of the gametes. However, the gametes present the potency to give rise to the next generation.

Artificial reprogramming occurs when biologists cultivate in vitro human ECS (hECS) or when differentiated cells are reprogramed by a pool of factors to produce human induced pluripotent stem cells (hiPSC). The purpose of the next chapters is to screen for known epigenetic status of the Alu and protective mechanisms against their activation in hiPSC and hECS as well as in the zygote and the PGCs.

\section{Epigenetic status of Alu during induced-pluripotency}

In 2007, a scientific revolution happens when Takahashi et al. successfully induced hiPSC from adult human fibroblasts by retrovirus-mediated transfection of the transcription factors Oct3/4, Sox2, Klf4, and c-Myc [52]. In 2011, different searchers de-differentiated fibroblasts into hiPSC using the Takahashi method. These authors observed numerous genetic defaults affecting the hiPSC, somatic mutations [53], copy number variations [54, 55], tumorigenicity [56] and epigenetic abnormalities [57]. Some years later, three studies converged and reported activations of transposons during the induced reprogramming performed to produce iPSCs [58-60].

Klawitter et al.began by collecting hiPSC and their matched parental cells. RT-qPCR revealed increased 
transcription of L1 containing the essential components of the retro-transposition machinery used by Alu (ORF1 and ORF2), whereas the L1 promoter was de-methylated according to bisulfite DNA sequencing. Then, they applied retrotransposon capture sequencing (RC-seq) to map the genomic integration sites of retrotransposon insertions. The results revealed increased transposition events both after reprogramming in hiPSC and during culture of hESC with an average of 1411 new Alu insertion per sample analyzed. $83 \%$ of all transposons insertions not previously referenced involved Alu, among which $88 \%$ of Alu concern the AluYa5 subfamily. The authors defined de novo insertion as not found in the parental matched cells, not previously referenced and not found in an earlier hESC passage or in multiple hiPSC or hESC lines. Among all de novo insertions, PCR validates eight L1, seven Alu and two SVA. That study demonstrates L1 and Alu retro-transposition in hiPSC apparently triggered by the induced reprogramming [58].

Thus, artificial reprogramming producing iPSCs increased deleterious Alu transposition compromising the high expectation for regenerative medicine. Of note, the retrovirus that transduced the reprogramming factors may well affect the endogenous retrotransposition activity found in hiPSCs, as explained later. However, the increased transposition detected in the cells reprogrammed by the transduced factors triggers the question of how the cells protect themselves from transposition during reprogramming in vivo and naturally.

\section{Epigenetics of Alu and reprogramming the zygote}

The first wave of reprogramming starts in the zygote and ends once the blastocyst implants in the uterus. All cells composing the pre-implanted embryo that derived from the fertilized egg were reprogrammed. The methylation dynamic of Alu was indirectly studied during reprogramming of the zygote in human [61-63]. In those works, sperm and oocytes are collected from free-informed consent and healthy men and women donors. Then, intracytoplasmic sperm injection (ICSI) is performed on metaphase II oocytes to obtain zygotes. Some searchers also isolate both pronuclei in the zygote to study their demethylation processes separately. Morula are obtained at different stages in vitro (two cells, four cells, and so on) up to a blastocyst. Postimplanted embryos came from aborted fetuses. By following that research strategy, searchers were able to acquire human methylome data in all these successive cells, using bisulfite-sequencing techniques, producing datasets used to recapitulate DNA methylation dynamic during the zygote reprogramming process. Globally, data generated support enrichment of methylation in Alu and across the different development stages (Fig. 2).
As shown in the Supplemental Fig. 11 of the Smith et al. publication, three types of transposons (IAP, LINE containing the L1, and SINE containing Alu) most highly methylated in the sperm as compared with the zygote and the oocyte were more stable in term of methylation across cleavages in the morula [63]. This was consistent with the work of Guo et al. The later recorded complete human methylomes encompassing the embryonic reprogramming using both reduced representation bisulphite sequencing (RRBS) and whole-genome bisulphite sequencing (bs-seq) approaches [61]. Guo et al. results revealed global methylation of the DNA decreasing gradually from $41 \%$ genome-wide in the 2 cell morula to $29 \%$ in the inner cell mass (ICM) of the embryo, whereas in Alu sequences, the methylation levels obtained remains higher (around 50\%) and more stable. This means that a bulk of methylation remains in the Alu repeats during the reprograming of the zygote, as observed in at least two independent works (Fig. 3).

In 2018, Zhu et al. provided similar tracing of the methylome changes across the first wave of zygote reprogramming at the single cell level, recapitulating more precisely the behavior of DNA methylation within Alu [62]. According to the results obtained, Alu strongly de-methylate from the late zygote to the two-cell stage as well as from the eight-cell to the morula stage. However, Alu and L1 enriched for de novo methylated $\mathrm{CpG}$ sites during both transitions from the early male pronuclear to the mid-pronuclear stage, as well as from the four to eight cells containing morula. According to the authors, de novo methylation favors Alu, most likely repressing their transcriptional activity to avoid mobilization and genome instability. Finally, the review of Greenberg claims that the highest retention of DNA methylation occurs in young and potentially active transposable elements, mentioning the SINE-VNTR-Alu (SVA) hominid-specific retrotransposon [64].

Thus, all the different studies converge for increased methylation once the embryos are implanted. The results obtained at the single cell resolution showed that the apparently stable methylation of Alu as seen in bulk samples and coinciding in time with a decreased methylation genomewide in the developing zygote seems highly dynamic, and involves global demethylation and focal re-methylation. Further research on RNA-mediated silencing of Alu during the reprogramming of the zygote is strongly required. Indeed, germline specific RNAs may well control Alu transposition during precise time windows in which Alu are de-methylated. This could also explain focal re-methylation within Alu. That kind of mechanism was described for germline specific RNAs able to control retrotransposons during the reprogramming of the germ cells in plants, named the RNA-directed DNA methylation, silencing 
Fig. 2 De-methylation and activation of L1-dependent Alu retrotransposon across reprogramming, de-differentiation, viral infections, cancers and brain mosaicism

\section{Zygotic reprogramming}

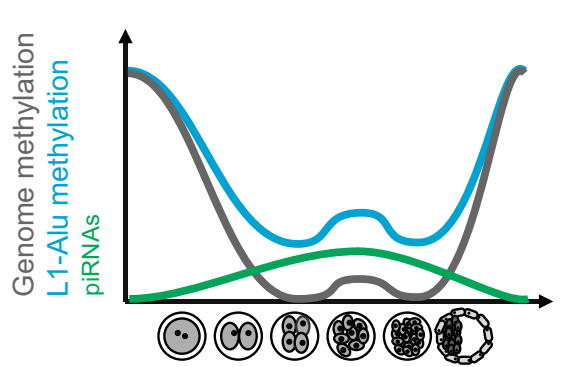

De-differentiation

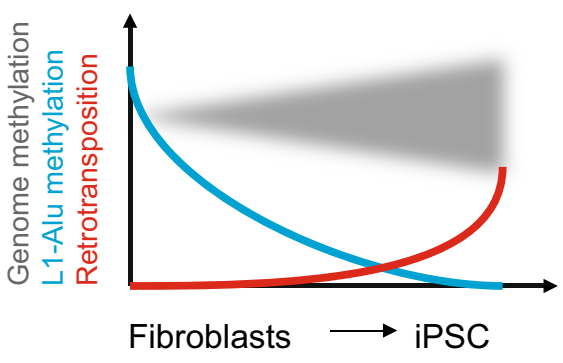

Cancers

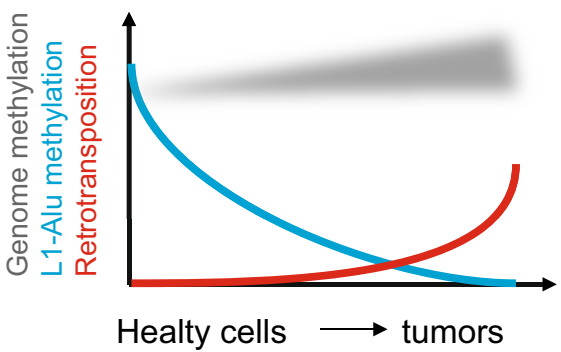

PGCs reprogramming

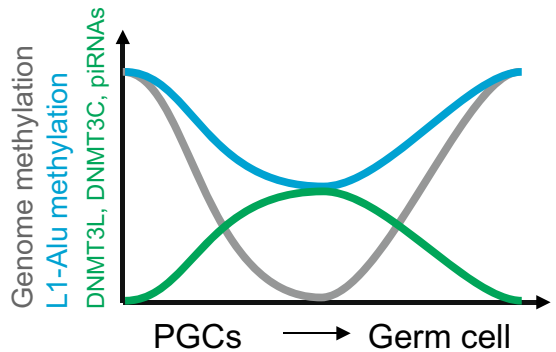

Viral infection

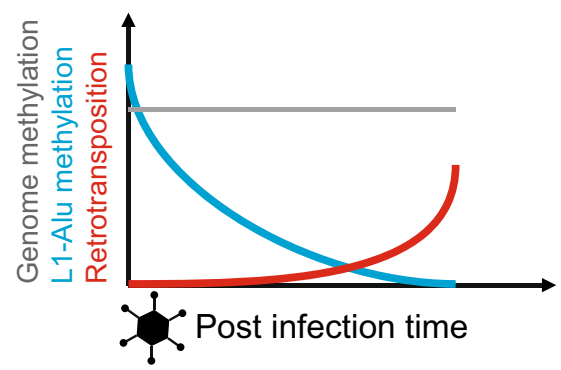

Brain mosaicism

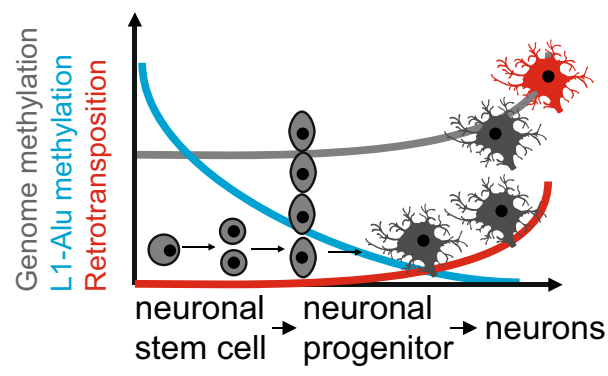

transposons, but the existence of that pathway in human seems unknown [65].

\section{Epigenetics status of Alu during the primordial germ cell reprogramming}

The second reprogramming wave affects the primordial germ cells (PGC). PGCs are the precursor of the germ cell lineage and are reprogrammed when they colonized the gonads during the embryonic development. PGC reprogramming persists during the sexual differentiation process in the developing embryo. Re-methylation begins in the malederived prospermatogonia or gonocytes and after birth in the female-derived growing oocytes. Note that most works performed on PGCs reprogramming were done in mouse lacking the dimeric primates specific Alu. Therefore, transposition of the knowledge from mouse to human is difficult.
In rodents PGCs, transposons bypass partly the reprogramming methylated by germline-cell specific DNMT3L and DNMT3C [66, 67]. The DNA methyl-transferases (DNMTs) consist of a family of enzymes able to transfer a methyl group from the S-Adenosyl Methionine (SAM) to the cytosine in the DNA, thus responsible for DNA methylation. The family contains the DNMT1 responsible for the maintenance of methylation across cell division, the DNMT3-A and -B that are able to methylate DNA de novo and both DNMT3L catalyzer, which is not able to methylate DNA alone but increased the activity of other DNMTs, as well as the germline-specific DNMT3C. DNMTs are essential for embryonic survival. DNMT3C protects male germ cells from transposon activity during reprogramming [66], whereas mice deleted for DNMT3L were sterile with adult males lacking spermatozoids as a consequence of a 'catastrophic' meiosis [67]. 
A

Methylated Alu

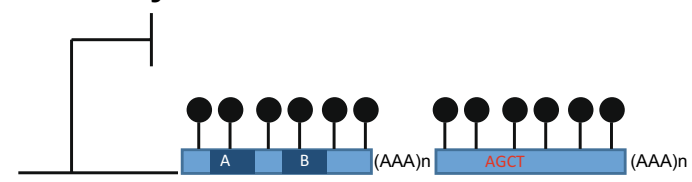

De-methylated Alu

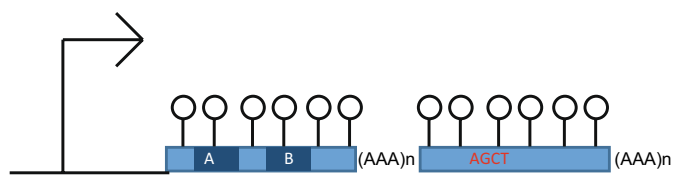

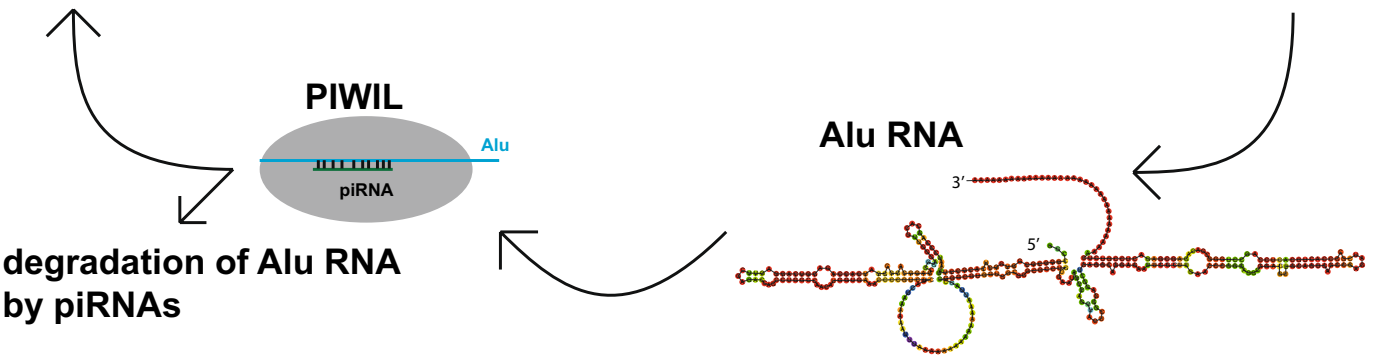

B

Neurofibromatosis,

Alu insertion in intron of NF1 producing a mature RNA lacking exon 6
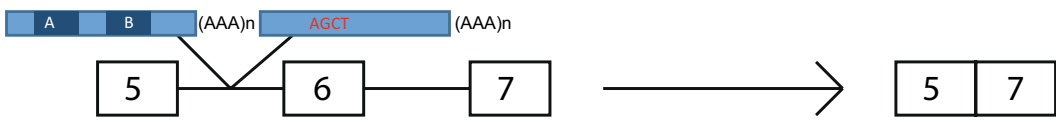

Hemophilia B,

Alu insertion in exon 4 of Factor IX creating a premature Stop

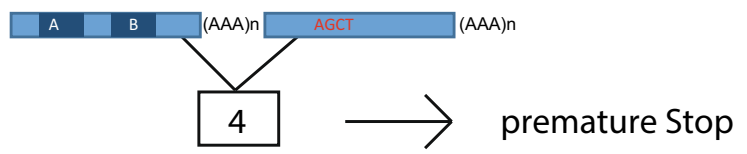

Breast cancer,

Deletion by recombination of Alu elements located between exons 15 and 16 in BRCA1

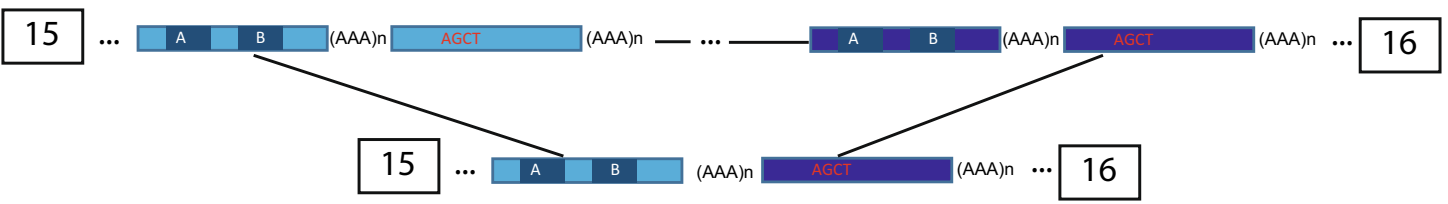

Fig. 3 Mechanisms controlling Alu activities and examples of Alu in Human diseases. a Epigenetic and post-transcriptional mechanisms controlling Alu activity. DNA methylation prevent Alu transcription. In case of de-methylated Alu such as during reprograming, Alu RNAs could be post-transcriptionally controlled by P-elementinduced wimpy testis (PIWI)-interacting RNAs (piRNAs). The piRNAs control retro-transposition by destroying the RNA encoded by

Importantly, in addition to DNA methylation, a subclass of the small non-coding RNAs also repress transpositions during PGCs reprogramming named the P-element-induced
Alu within the piRNA-induced silencing complexes (piRISC). In addition, piRNAs and may also help for de novo DNA methylation of Alu. b Role of Alu is developmental diseases. Three examples that are discussed in the text are schematized. First, an Alu insertion within an intron causing neurofibromatosis. Second, an Alu insertion within an exon causing Hemophilia. Third, rearrangement in an Alu rich region of BRCA1 important for breast cancers

wimpy testis (PIWI)-interacting RNAs (piRNAs). The piRNAs are germline specific RNAs which main function is to control retro-transposition by destroying the RNA encoded 
by retrotransposons. The piRNAs may also help for de novo DNA methylation at some retrotransposon promoters [68]. A proof of principle in human cells cultivated in vitro revealed that the microprocessor (Drosha-DGCR8) negatively regulates LINE-1 and Alu retro-transposition [69].

\section{Alu and imprinting}

In human, 30 to 200 genes may be imprinted (http://www. geneimprint.org). These imprinted genes present a somatic lifelong mono-allelic expression inversely correlated with a mono-allelic methylation status that depends on the parental origin of the alleles. The imprinting was thought to occur during gametogenesis, a period when both maternal and paternal genomes are clearly separated [70]. The silenced methylated allele should resist the zygotic reprogramming. In other words, paternal and maternal imprinted genes contain an allele deriving from either the sperm or the ovum pronuclei that bypassed the reprogramming and that are kept methylated and silenced lifelong. For example, PEG3, PEG10, PEG13, SNRPN, IGF2 and RB1 are imprinted paternally expressed genes, whereas MEG1, H19 and IGF2R are imprinted and maternally expressed genes.

The question emerged whether Alu and other transposons are also able to bypass reprogramming by using the imprinting mechanism. In the single cells methylome study of Zhu et al., differentially methylated regions (DMRs) were analyzed between oocytes and sperm [62]. The authors reported that all "31 known imprinting controls regions (ICR)" were included among these DMRs. When analyzing the distribution of these DMRs in the genome, they found strong enrichment in Alu elements. However, when John M. Greally studied the genomic composition in imprinted versus non-imprinted loci, he reported that imprinted regions lack Alu [71]. Thus, both parental gametes strongly differ in term of DNA methylation in both imprinted loci and Alu repeats without co-localization between them. Paradoxically, some imprinted regions were reported to be derived from previous transposition events, whereas genomic imprinting may have evolved from a defense mechanism against transposable elements depending on DNA methylation established in germ cells [72].

\section{Alu epigenetics and human infertility}

The epigenetics control of Alu during both reprogramming waves and Alu involvement in the imprinting process are essential for the proper development of both the germ cells and the zygote. Thus, epigenetic defaults in Alu could explain at least some cases of infertility. Infertility in human was arbitrary defined by the world health organization (WHO) as the absence of pregnancy after one year of regular unprotected sexual intercourse in a couple. Even if arbitrary, the definition influences the access to assisted reproductive technologies (ART) for infertile couples. Epigenetics of Alu in the germ cells seems of high concern to understand the etiology of unexplained infertility [73], but also in child conceived with ART, as the incidence of rare imprinting disorders was increased in ART conceived children [74]. Currently, Alu methylation in germ cells of infertile men is expanding, but remains rarely, if not at all studied in the oocytes of infertile women. In general, the contribution of Alu in both men and women infertility remain largely understudied.

In an ongoing collaborative and unpublished study on DNA methylation in sperm of infertile men, for which half present normal sperm parameters, we recently identified demethylation of Alu by using a genome-wide approach and are actually assessing to replicate these results. These results were at the origin of my interest in the study of Alu. Urdinguio et al. reported compatible results: decreased cytosine methylation in Alu, notably the Yb8 in their work, in the sperm of men suffering from unexplained infertility [75]. In addition, Alu may also probably contribute to female infertility. One line of evidence concern NLRP7. Apparently, the correct gene copy of NLRP7 is required for the female reproductive contribution. Indeed, large homozygous deletion mutations identified in NLRP7 were responsible for deficient pregnancies and reproductive wastage [76], whereas the breakpoints are located within Alu repeats [77].

\section{Alu and mosaicism}

Mosaicism implies the existence of two or more cells with different genotypes present within one individual and deriving from the same zygote. Alu retro-transposition affecting some embryonic cells may create mosaicism in the organism. The early embryo is the primary niche for the accumulation of new retrotransposition. Of importance, somatic mosaicism created by retrotransposition should not be heritable in contrast to retrotransposition affecting the germline. Pathogenic mosaicism caused by Alu retrotransposition in Human has already been reported [78]. In that work, multiple complex rearrangement sequences encompassing exon 1 of GAN were described for a patient suffering Giant axonal neuropathy. The patient's genome(s) was analyzed by quantitative real-time PCR and breakpoint DNA sequencing. The authors identified micro-homology of Alu sequences at the breakpoint suggesting Alu mediated recombination [78].

However, human Alu mediated mosaicism remain poorly studied as compared with mosaicism involving L1 retrotransposition in rodents, especially in neuronal cells [79]. The following key biological aspects of mosaicism induced by retro-transposition strongly required to be confirmed in the humans Alu context. First, retrotransposons can mobilize 
both during embryogenesis as well as during division in the neuronal lineage causing somatic genome mosaicism. Second, the neuronal progenitor cells and post-mitotic neurons accommodate retrotransposition in contrast to other cell lineages. Finally, retrotransposition clearly occurs in the brain and creates neuronal diversity.

Today, we believe that the brain is mosaic [80]. The brain contains approximately 80 billion neurons derived from a colossal number of progenitor's division. Thus, even if rare, retro-transpositions or wrongly repaired DNA errors could statically occur during that enormous amount of replication in neuronal progenitors, and are thought to participate in the brain mosaicism. Supporting that hypothesis, endogenous retro-elements have been used to successfully trace numerous lineage and sub-lineages of neuronal cells by Evrony et al., using whole-genome sequencing at the single neuron resolution [81]. The profiling of L1-transposon insertion suggested that 0.2 to $1 \mathrm{~L} 1$ insertion may occur per "neuronal genome" [80]. Actually, new evidences suggest that pathogenic transposition contributes to a broad range of neurological diseases which etiology remains poorly understood [82].

\section{Viral contribution to Alu transposition boom}

Evidences suggest that Alu may also have originated from reverse transcribed 7SL RNA in a viral infected host. A first evidence supporting a viral origin of Alu concerns the detection of reverse transcription of 7SL RNA in a quail cell line infected by a deficient avian Rous sarcoma virus [83]. The authors demonstrated that 7SL RNA may serve as a template for reverse transcription in the virion with transfer RNA (tRNA) used as primer and resulting in a cDNA of $135 \mathrm{bp}$ compatible in size with an Alu monomer. Unfortunately, the cDNA was not sequenced. Interestingly, the host 7SL RNA increased in cells infected by virus, later quantified approximately at 12 copy per retroviral particles [84].

The viral origin of Alu seems difficult to study, but recent evidences emerge reporting increased Alu retrotransposition in viral infected cells. One report experimentally demonstrates increased Alu transcription, transposition and copy in primary CD4 + cells infected by HIV-1 [85]. In 1995, Russanova et al.isolated nucleus of HeLa cells infected with adenovirus at 25, 50 and 150 plaque-forming units (PFU) compared with nuclei of uninfected cells. They developed an original method combining nuclear run-on and RNase $\mathrm{H}$ assay to assess how Alu are expressed. Their results revealed that Alu elements were masked from the Pol III transcriptional machinery in the nuclei of uninfected cells, whereas the Pol III machinery actively transcribed Alu in infected cell nuclei. The authors concluded that Alu repeats are efficiently sequestered by chromatin proteins and that adenovirus infection partially overrides that repressive mechanism
[86]. Thus, the contribution of different virus boosting Alu transposition during evolution is credible.

\section{Innate immune response and Alu}

The innate immune system is an evolutionary conserved host defense system acting against retrovirus, but also against the activation of retrotransposon [87]. In contrast to a retrovirus, which assembles itself and buds out the infected cell before infecting other cells, Alu and other retrotransposons stay within the host cell. However, Alu and other retrotransposons are present as DNA sequences in all human cell's and may add a new DNA copy of them transmissible to all cells constituting the next generation in case the retro-transposition affects the germline. Once retrotransposons such as Alu are expressed in a cell, for example, during a viral infection [86], various nucleic acid forms of Alu may theoretically appear, namely (1) single stranded RNA (ssRNS), (2) RNA:RNA duplex or double stranded RNA (dsRNA), (3) RNA:DNA heteroduplex, and (4) DNA:DNA duplex or double stranded DNA (dsDNA). Apparently, both RNA:RNA and DNA:DNA Alu duplex may appear in the cytoplasm as intermediates and may be sensed by host cell derived molecular sensors. The melanoma differentiation-associated protein 5 (MDA5) detects the presence of RNA:RNA Alu [88]. It remains unknown if the cyclic GMP-AMP synthase (cGAS) detects DNA:DNA Alu [89]. The innate immune system should tolerate a basal presence of Alu or it will trigger targeted nucleic acid destruction. In addition, the innate immune system may produce cytokines and inflammation, useful to fight the virus, but useless in case of within-cell restricted retrotransposon, excepted in the case of a transposoninduced cancer. In fact, the immune system has to tolerate domesticated Alu, balancing between recognition of self and activation of innate immunity. Indeed, if the innate immune system acts too strongly to Alu, it may eventually give rise to autoimmune disorders [90].

Concerning nucleic acid forms, first, ssRNA Alu is produced by Pol III-dependent transcription [21] and is destroyed by Dicer1 [91]. In case of geographic atrophy, a common cause of blindness in industrialized countries, Dicer is deficient and increased ssRNA Alu toxicity is detectable in the eyes [91]. Alu RNA mediated toxicity affects more precisely cells constituting the retinal pigment epithelium [92]. Second, Alu RNA-DNA heteroduplex may be produced during reverse transcription as a common feature of retrotransposons, as well as DNA:DNA duplex required for the integration of a new copy of the transposon [87]. In addition to these forms, Alu RNA:RNA duplex has been identified [88] deriving from sense combined with antisense transcription [87]. The most convincing evidence supporting the involvement of Alu in innate immune response came 
from a study by Ahmad et al. on dsRNA sensor MDA5 in Aicardi-Goutières syndrome (AGS), an inherited encephalopathy [88]. Alu RNA:RNA hybrids formed by inverted Alu activate the MDA5 mutated gene found in AGS but not wild-type MDA5. The authors reported that in AGS, Alu RNA:RNA duplex was the primary ligands triggering aberrant MDA5-mediated antiviral signaling [88]. MDA5 was known as a cytosolic innate immune sensor of dsRNA generated during viral replication [93].

One report discovered that Alu may modulate autoimmunity in case of systemic lupus erythematosus (SLE). SLE is an autoimmune disease caused by the production of autoantibodies targeting the protein Ro60. When Hung et al. aim to identify RNAs bound to Ro60 using individual nucleotide crosslinking and immunoprecipitation (iCLIP) followed by sequencing, they discovered Alu RNA bounded to Ro60 [94]. These Alu RNAs were increased in the blood of patients suffering SLE as compared to controls. Alu RNAs and interferon-regulated genes increased also in the absence of the Ro60 gene in cell culture, as well as in the presence of interferon alpha (IFN $\alpha$ ). Thus, Alu RNA levels were linked to the Ro60 autoantibody responses in SLE and may probably contribute to human autoimmunity. Finally, according to Herbert, Alu may regulate inflammation based on their potential to form various conformations, enabling a rapid reprogramming of cellular pathways. Alu may induce so called non-B-DNA conformations named Z-DNA, triplexes and quadruplexes. These particular conformations modified the way the genome is expressed [95].

\section{Conclusion}

Alu retrotransposons are involved in fundamental biological processes (Fig. 2). In addition, Alu may contribute to numerous genetic diseases with apparently unexplained etiologies. The clinical impact of Alu is probably underestimated, as Alu remain badly understood in the medical genetic and clinical context. The physiology of Alu retrotransposition remains to be better studied, as the mechanism simplified in the present review is still not completely understood at the molecular level. Numerous questions remain open concerning Alu. During reprogramming, it would be interesting to know if RNA interference controls Alu during precise time windows in which Alu are de-methylated in the zygote. The interaction between piRNAs and de novo methylation in the germ cells is possible but unknown in human. That putative interaction could provide a better understanding of how reprogramming did not allow massive retrotransposition. Concerning the involvement of Alu in infertility, it would be of great interest to study the epigenetic status of the Alu that are located in the NLRP7 locus in the oocytes of woman suffering miscarriages, as well as the methylation of Alu in the sperm of men suffering unexplained infertility. How to explain the paradox that imprinted regions significantly lacks Alu repeats, whereas imprinting may be derived from previous transposition events? Considering that virus may "wake up" Alu, what would be the impact of the actual pandemic coronavirus on the Alu mediated evolution of our genomes? In the innate immunity, are Alu DNA:DNA detected in the cytoplasm by cGAS? Does Alu retrotransposition occur in the neuronal lineage creating mosaicism as found for L1 retrotransposition? Thus, numerous studies still may be conducted on the fascinating L1-dependant and Pol III transcribed Alu retrotransposon.

Funding Open Access funding provided by Université de Genève. Swiss Centre for Applied Human Toxicology (SCAHT).

\section{Declarations}

Conflict of interest I have no competing interests.

Open Access This article is licensed under a Creative Commons Attribution 4.0 International License, which permits use, sharing, adaptation, distribution and reproduction in any medium or format, as long as you give appropriate credit to the original author(s) and the source, provide a link to the Creative Commons licence, and indicate if changes were made. The images or other third party material in this article are included in the article's Creative Commons licence, unless indicated otherwise in a credit line to the material. If material is not included in the article's Creative Commons licence and your intended use is not permitted by statutory regulation or exceeds the permitted use, you will need to obtain permission directly from the copyright holder. To view a copy of this licence, visit http://creativecommons.org/licenses/by/4.0/.

\section{References}

1. Houck CM, Rinehart FP, Schmid CW (1979) A ubiquitous family of repeated DNA sequences in the human genome. J Mol Biol 132(3):289-306

2. Umylny B, Presting G, Efird JT, Klimovitsky BI, Ward WS (2007) Most human Alu and murine B1 repeats are unique. J Cell Biochem 102(1):110-121

3. Deininger PL, Batzer MA (1999) Alu repeats and human disease. Mol Genet Metab 67(3):183-193

4. Deininger P (2011) Alu elements: know the SINEs. Genome Biol 12(12):236

5. Bennett EA, Keller H, Mills RE, Schmidt S, Moran JV, Weichenrieder O et al (2008) Active Alu retrotransposons in the human genome. Genome Res 18(12):1875-1883

6. Ivanova E, Berger A, Scherrer A, Alkalaeva E, Strub K (2015) Alu RNA regulates the cellular pool of active ribosomes by targeted delivery of SRP9/14 to 40S subunits. Nucleic Acids Res 43(5):2874-2887

7. Weiner AM (1980) An abundant cytoplasmic 7S RNA is complementary to the dominant interspersed middle repetitive DNA sequence family in the human genome. Cell 22(1):209-218

8. Ullu E, Murphy S, Melli M (1982) Human 7SL RNA consists of a 140 nucleotide middle-repetitive sequence inserted in an alu sequence. Cell 29(1):195-202 
9. Quentin Y (1992) Fusion of a free left Alu monomer and a free right Alu monomer at the origin of the Alu family in the primate genomes. Nucleic Acids Res 20(3):487-493

10. Kriegs JO, Churakov G, Jurka J, Brosius J, Schmitz J (2007) Evolutionary history of 7SL RNA-derived SINEs in Supraprimates. Trends Genet 23(4):158-161

11. Batzer MA, Deininger PL (2002) Alu repeats and human genomic diversity. Nat Rev Genet 3(5):370-379

12. Ahmed M, Li W, Liang P (2013) Identification of three new Alu $\mathrm{Yb}$ subfamilies by source tracking of recently integrated $\mathrm{Alu} \mathrm{Yb}$ elements. Mobile DNA 4(1):25

13. Schmid CW (1991) Human Alu subfamilies and their methylation revealed by blot hybridization. Nucleic Acids Res 19(20):5613-5617

14. Batzer MA, Deininger PL, Hellmann-Blumberg U, Jurka J, Labuda D, Rubin CM et al (1996) Standardized nomenclature for Alu repeats. J Mol Evol 42(1):3-6

15. Feusier J, Watkins WS, Thomas J, Farrell A, Witherspoon DJ, Baird L et al (2019) Pedigree-based estimation of human mobile element retrotransposition rates. Genome Res 29(10):1567-1577

16. Cordaux R, Hedges DJ, Herke SW, Batzer MA (2006) Estimating the retrotransposition rate of human Alu elements. Gene 373:134-137

17. Xing J, Zhang Y, Han K, Salem AH, Sen SK, Huff CD et al (2009) Mobile elements create structural variation: analysis of a complete human genome. Genome Res 19(9):1516-1526

18. Jurka J, Smith T (1988) A fundamental division in the Alu family of repeated sequences. Proc Natl Acad Sci USA 85(13):4775-4778

19. Martinez-Gomez L, Abascal F, Jungreis I, Pozo F, Kellis M, Mudge JM et al (2020) Few SINEs of life: Alu elements have little evidence for biological relevance despite elevated translation. NAR Genomics Bioinform 2(1):023

20. Konkel MK, Walker JA, Hotard AB, Ranck MC, Fontenot CC, Storer J et al (2015) Sequence analysis and characterization of active human Alu subfamilies based on the 1000 genomes pilot project. Genome Biol Evol 7(9):2608-2622

21. Conti A, Carnevali D, Bollati V, Fustinoni S, Pellegrini M, Dieci G (2015) Identification of RNA polymerase III-transcribed Alu loci by computational screening of RNA-Seq data. Nucleic Acids Res 43(2):817-835

22. Ferrari R, de Llobet Cucalon LI, Di Vona C, Le Dilly F, Vidal E, Lioutas A et al (2020) TFIIIC binding to Alu elements controls gene expression via chromatin looping and histone acetylation. Mol Cell 77(3):475-87.e11

23. Christian CM, deHaro D, Kines KJ, Sokolowski M, Belancio VP (2016) Identification of L1 ORF2p sequence important to retrotransposition using Bipartile Alu retrotransposition (BAR). Nucleic Acids Res 44(10):4818-4834

24. Srikanta D, Sen SK, Conlin EM, Batzer MA (2009) Internal priming: an opportunistic pathway for L1 and Alu retrotransposition in hominins. Gene 448(2):233-241

25. Gruber AR, Lorenz R, Bernhart SH, Neubock R, Hofacker IL (2008) The Vienna RNA websuite. Nucleic Acids Res 36(Web Server issue):W70-W74

26. Esnault C, Casella J-F, Heidmann T (2002) A Tetrahymena thermophila ribozyme-based indicator gene to detect transposition of marked retroelements in mammalian cells. Nucleic Acids Res 30(11): 49

27. Dewannieux M, Esnault C, Heidmann T (2003) LINE-mediated retrotransposition of marked Alu sequences. Nat Genet 35(1):41-48

28. Schmid CW (2003) Alu: a parasite's parasite? Nat Genet $35(1): 15-16$
29. Wallace MR, Andersen LB, Saulino AM, Gregory PE, Glover TW, Collins FS (1991) A de novo Alu insertion results in neurofibromatosis type 1. Nature 353(6347):864-866

30. Vidaud D, Vidaud M, Bahnak BR, Siguret V, Gispert Sanchez S, Laurian Y et al (1993) Haemophilia B due to a de novo insertion of a human-specific Alu subfamily member within the coding region of the factor IX gene. Eur J Hum Genet 1(1):30-36

31. Nicholls RD, Fischel-Ghodsian N, Higgs DR (1987) Recombination at the human $\alpha$-globin gene cluster: sequence features and topological constraints. Cell 49(3):369-378

32. Lehrman MA, Goldstein JL, Russell DW, Brown MS (1987) Duplication of seven exons in LDL receptor gene caused by Alu-Alu recombination in a subject with familial hypercholesterolemia. Cell 48(5):827-835

33. Wang Y, Bernhardy AJ, Nacson J, Krais JJ, Tan Y-F, Nicolas E et al (2019) BRCA1 intronic Alu elements drive gene rearrangements and PARP inhibitor resistance. Nat Commun 10(1):5661

34. Larsen PA, Hunnicutt KE, Larsen RJ, Yoder AD, Saunders AM (2018) Warning SINEs: Alu elements, evolution of the human brain, and the spectrum of neurological disease. Chromosome Res 26(1-2):93-111

35. Liu W-M, Maraia RJ, Rubin CM, Schmid CW (1994) Alu transcripts: cytoplasmic localisation and regulation by DNA methylation. Nucleic Acids Res 22(6):1087-1095

36. Liu W-M, Schmid CW (1993) Proposed roles for DNA methylation in Alu transcriptional repression and mutational inactivation. Nucleic Acids Res 21(6):1351-1359

37. Xiang S, Liu Z, Zhang B, Zhou J, Zhu B-D, Ji J et al (2010) Methylation status of individual CpG sites within Alu elements in the human genome and Alu hypomethylation in gastric carcinomas. BMC Cancer 10:44

38. Zhou W, Liang G, Molloy PL, Jones PA (2020) DNA methylation enables transposable element-driven genome expansion. Proc Natl Acad Sci USA 117(32):19359-19366

39. Varshney D, Vavrova-Anderson J, Oler AJ, Cairns BR, White RJ (2015) Selective repression of SINE transcription by RNA polymerase III. Mobile Genetic Elem 5(6):86-91

40. Varshney D, Vavrova-Anderson J, Oler AJ, Cowling VH, Cairns BR, White RJ (2015) SINE transcription by RNA polymerase III is suppressed by histone methylation but not by DNA methylation. Nat Commun 6(1):6569

41. Zhang W, Edwards A, Fan W, Deininger P, Zhang K (2011) Alu distribution and mutation types of cancer genes. BMC Genomics $12: 157$

42. Slebos RJ, Resnick MA, Taylor JA (1998) Inactivation of the p53 tumor suppressor gene via a novel Alu rearrangement. Cancer Res 58(23):5333-5336

43. Shih C, Weinberg RA (1982) Isolation of a transforming sequence from a human bladder carcinoma cell line. Cell 29(1):161-169

44. Ye D, Jiang D, Zhang X, Mao Y (2020) Alu methylation and risk of cancer: a meta-analysis. Am J Med Sci 359(5):271-280

45. Horvath S (2013) DNA methylation age of human tissues and cell types. Genome Biol 14(10):R115

46. Unnikrishnan A, Hadad N, Masser DR, Jackson J, Freeman WM, Richardson A (2018) Revisiting the genomic hypomethylation hypothesis of aging. Ann N Y Acad Sci 1418(1):69-79

47. Buj R, Mallona I, Diez-Villanueva A, Barrera V, Mauricio D, Puig-Domingo M et al (2016) Quantification of unmethylated Alu (QUAlu): a tool to assess global hypomethylation in routine clinical samples. Oncotarget 7(9):10536-10546

48. Mustafina OE (2013) The possible roles of human Alu elements in aging. Front Genet 4:96

49. Lopatina N, Haskell JF, Andrews LG, Poole JC, Saldanha S, Tollefsbol T (2002) Differential maintenance and de novo 
methylating activity by three DNA methyltransferases in aging and immortalized fibroblasts. J Cell Biochem 84(2):324-334

50. Reik W, Dean W, Walter J (2001) Epigenetic reprogramming in mammalian development. Science 293(5532):1089-1093

51. Mitalipov S, Wolf D (2009) Totipotency, pluripotency and nuclear reprogramming. Adv Biochem Eng Biotechnol 114:185-199

52. Takahashi K, Tanabe K, Ohnuki M, Narita M, Ichisaka T, Tomoda $\mathrm{K}$ et al (2007) Induction of pluripotent stem cells from adult human fibroblasts by defined factors. Cell 131(5):861-872

53. Gore A, Li Z, Fung HL, Young JE, Agarwal S, Antosiewicz-Bourget $\mathrm{J}$ et al (2011) Somatic coding mutations in human induced pluripotent stem cells. Nature 471(7336):63-67

54. Hussein SM, Batada NN, Vuoristo S, Ching RW, Autio R, Narva E et al (2011) Copy number variation and selection during reprogramming to pluripotency. Nature 471(7336):58-62

55. Laurent LC, Ulitsky I, Slavin I, Tran H, Schork A, Morey R et al (2011) Dynamic changes in the copy number of pluripotency and cell proliferation genes in human ESCs and iPSCs during reprogramming and time in culture. Cell Stem Cell 8(1):106-118

56. Ben-David U, Benvenisty N (2011) The tumorigenicity of human embryonic and induced pluripotent stem cells. Nat Rev Cancer 11(4):268-277

57. Lister R, Pelizzola M, Kida YS, Hawkins RD, Nery JR, Hon G et al (2011) Hotspots of aberrant epigenomic reprogramming in human induced pluripotent stem cells. Nature 471(7336):68-73

58. Klawitter S, Fuchs NV, Upton KR, Munoz-Lopez M, Shukla R, Wang J et al (2016) Reprogramming triggers endogenous L1 and Alu retrotransposition in human induced pluripotent stem cells. Nat Commun 7:10286

59. Wissing S, Munoz-Lopez M, Macia A, Yang Z, Montano M, Collins W et al (2012) Reprogramming somatic cells into iPS cells activates LINE-1 retroelement mobility. Hum Mol Genet 21(1):208-218

60. Friedli M, Turelli P, Kapopoulou A, Rauwel B, Castro-Diaz N, Rowe HM et al (2014) Loss of transcriptional control over endogenous retroelements during reprogramming to pluripotency. Genome Res 24(8):1251-1259

61. Guo H, Zhu P, Yan L, Li R, Hu B, Lian Y et al (2014) The DNA methylation landscape of human early embryos. Nature 511(7511):606-610

62. Zhu P, Guo H, Ren Y, Hou Y, Dong J, Li R et al (2018) Single-cell DNA methylome sequencing of human preimplantation embryos. Nat Genet 50(1):12-19

63. Smith ZD, Chan MM, Mikkelsen TS, Gu H, Gnirke A, Regev A et al (2012) A unique regulatory phase of DNA methylation in the early mammalian embryo. Nature 484(7394):339-344

64. Greenberg MVC, Bourc'his D (2019) The diverse roles of DNA methylation in mammalian development and disease. Nat Rev Mol Cell Biol 20(10):590-607

65. Matzke MA, Mosher RA (2014) RNA-directed DNA methylation: an epigenetic pathway of increasing complexity. Nat Rev Genet 15(6):394-408

66. Barau J, Teissandier A, Zamudio N, Roy S, Nalesso V, Herault Y et al (2016) The DNA methyltransferase DNMT3C protects male germ cells from transposon activity. Science 354(6314):909-912

67. Bourc'his D, Bestor TH (2004) Meiotic catastrophe and retrotransposon reactivation in male germ cells lacking Dnmt3L. Nature 431(7004):96-99

68. Sarkar A, Volff JN, Vaury C (2017) piRNAs and their diverse roles: a transposable element-driven tactic for gene regulation? FASEB J 31(2):436-446

69. Heras SR, Macias S, Plass M, Fernandez N, Cano D, Eyras E et al (2013) The Microprocessor controls the activity of mammalian retrotransposons. Nat Struct Mol Biol 20(10):1173-1181

70. Barlow DP (1993) Methylation and imprinting: from host defense to gene regulation? Science 260(5106):309-310
71. Greally JM (2002) Short interspersed transposable elements (SINEs) are excluded from imprinted regions in the human genome. Proc Natl Acad Sci USA 99(1):327-332

72. Renfree MB, Suzuki S, Kaneko-Ishino T (2013) The origin and evolution of genomic imprinting and viviparity in mammals. Philos Trans R Soc Lond B Biol Sci 368(1609):20120151

73. Hamada A, Esteves SC, Nizza M, Agarwal A (2012) Unexplained male infertility: diagnosis and management. Int Braz $\mathrm{J}$ Urol 38(5):576-594

74. Hiura H, Okae H, Chiba H, Miyauchi N, Sato F, Sato A et al (2014) Imprinting methylation errors in ART. Reprod Med Biol 13(4):193-202

75. Urdinguio RG, Bayón GF, Dmitrijeva M, Toraño EG, Bravo C, Fraga MF et al (2015) Aberrant DNA methylation patterns of spermatozoa in men with unexplained infertility. Hum Reprod 30(5):1014-1028

76. Messaed C, Chebaro W, Di Roberto RB, Rittore C, Cheung A, Arseneau J et al (2011) NLRP7 in the spectrum of reproductive wastage: rare non-synonymous variants confer genetic susceptibility to recurrent reproductive wastage. J Med Genet 48(8):540-548

77. Reddy R, Nguyen NMP, Sarrabay G, Rezaei M, Rivas MCG, Kavasoglu A et al (2016) The genomic architecture of NLRP7 is Alu rich and predisposes to disease-associated large deletions. Eur J Hum Genet EJHG 24(10):1445-1452

78. Shi M, Chen X, Zeng L, Li Z, Liang D, Wu L (2020) The rare Alus element-mediated chimerism of multiple de novo complex rearrangement sequences in GAN result in giant axonal neuropathy. Clin Chim Acta 502:91-98

79. Faulkner GJ, Garcia-Perez JL (2017) L1 mosaicism in mammals: extent, effects, and evolution. Trends Genet 33(11):802-816

80. McConnell MJ, Moran JV, Abyzov A, Akbarian S, Bae T, CortesCiriano I et al (2017) Intersection of diverse neuronal genomes and neuropsychiatric disease: the brain somatic mosaicism network. Science. https://doi.org/10.1126/science.aal1641

81. Evrony GD, Lee E, Mehta BK, Benjamini Y, Johnson RM, Cai X et al (2015) Cell lineage analysis in human brain using endogenous retroelements. Neuron 85(1):49-59

82. Jonsson ME, Garza R, Johansson PA, Jakobsson J (2020) Transposable elements: a common feature of neurodevelopmental and neurodegenerative disorders. Trends Genet 36(8):610-623

83. Chen PJ, Cywinski A, Taylor JM (1985) Reverse transcription of 7S L RNA by an avian retrovirus. J Virol 54(2):278-284

84. Telesnitsky A, Wolin SL (2016) The host RNAs in retroviral particles. Viruses 8(8):235

85. Jones RB, Song H, Xu Y, Garrison KE, Buzdin AA, Anwar N et al (2013) LINE-1 retrotransposable element DNA accumulates in HIV1-infected cells. J Virol 87(24):13307-13320

86. Russanova VR, Driscoll CT, Howard BH (1995) Adenovirus type 2 preferentially stimulates polymerase III transcription of Alu elements by relieving repression: a potential role for chromatin. Mol Cell Biol 15(8):4282-4290

87. Kassiotis G, Stoye JP (2016) Immune responses to endogenous retroelements: taking the bad with the good. Nat Rev Immunol 16(4):207-219

88. Ahmad S, Mu X, Yang F, Greenwald E, Park JW, Jacob E et al (2018) Breaching self-tolerance to Alu duplex RNA underlies MDA5-mediated inflammation. Cell 172(4):797-810.e13

89. Gao D, Wu J, Wu Y-T, Du F, Aroh C, Yan N et al (2013) Cyclic GMP-AMP synthase is an innate immune sensor of HIV and other retroviruses. Science 341(6148):903-906

90. Visan I (2018) Alu element risk. Nat Immunol 19(3):206

91. Meister G (2011) Dicer leaps into view. Nature 471(7338):308-309

92. Kaneko H, Dridi S, Tarallo V, Gelfand BD, Fowler BJ, Cho WG et al (2011) DICER1 deficit induces Alu RNA toxicity in agerelated macular degeneration. Nature 471(7338):325-330 
93. del Toro DY, Wu B, Hur S (2015) MDA5-filament, dynamics and disease. Curr Opin Virol 12:20-25

94. Hung T, Pratt GA, Sundararaman B, Townsend MJ, Chaivorapol $\mathrm{C}$, Bhangale $\mathrm{T}$ et al (2015) The Ro60 autoantigen binds endogenous retroelements and regulates inflammatory gene expression. Science 350(6259):455-459
95. Herbert A (2020) ALU non-B-DNA conformations, flipons, binary codes and evolution. R Soc Open Sci 7(6):200222

Publisher's Note Springer Nature remains neutral with regard to jurisdictional claims in published maps and institutional affiliations. 